TRANSACTIONS OF THE

AMERICAN MATHEMATICAL SOCIETY

Volume 355, Number 7, Pages 2755-2769

S 0002-9947(03)03270-7

Article electronically published on February 25, 2003

\title{
ON THE SPECTRAL SEQUENCE CONSTRUCTORS OF GUICHARDET AND STEFAN
}

\author{
DONALD W. BARNES
}

\begin{abstract}
The concept of a spectral sequence constructor is generalised to Hopf Galois extensions. The spectral sequence constructions that are given by Guichardet for crossed product algebras are also generalised and shown to provide examples. It is shown that all spectral sequence constructors for Hopf Galois extensions construct the same spectral sequence.
\end{abstract}

\section{INTRODUCTION}

A. Guichardet [3] has given two constructions for a spectral sequence with $E_{2}^{p, q}=$ $H^{p}\left(G, H^{q}(B, X)\right)$ and target the Hochschild cohomology $H^{\bullet}\left(G \times{ }_{\alpha} B, X\right)$ of the crossed product algebra $A=G \times{ }_{\alpha} B$, where $G$ is a group, $B$ is an algebra, $\alpha$ is a representation of $G$ by automorphisms of $B$ and the multiplication in $A$ is given by

$$
(g, b)\left(g^{\prime}, b^{\prime}\right)=\left(g g^{\prime}, \alpha_{g^{\prime}}^{-1}(b) b^{\prime}\right) .
$$

Here, $X$ is a left and right $A$-bimodule or, equivalently, a left module over $A^{e}=$ $A \otimes A^{\text {op }}$ where $A^{\text {op }}$ is the opposed algebra of $A$. These constructions are analogous to the Hochschild-Serre constructions for the spectral sequence of a group extension. He has asked if the methods of Barnes 1] can be used to show that they construct the same spectral sequence.

D. Stefan [5] has given a spectral sequence, based on the Grothendiek composite functor spectral sequence, for the cohomology of a Hopf Galois extension.

The three contexts have some features in common. All have a "large" algebra $A$ and the category $\mathcal{A}$ of $A$-(bi)modules, a subalgebra $B$, a "small" algebra $C$ which plays the role of a quotient of $A$ by $B$ and the category $\mathcal{C}$ of $C$-modules and a category $\mathcal{D}$ in which the filtered cochain complexes are constructed. All have a left exact functor $\phi: \mathcal{A} \rightarrow \mathcal{C}$ and a left exact functor $\psi: \mathcal{C} \rightarrow \mathcal{D}$, and the spectral sequences have as target the right derived functors of the composite $\theta=\psi \circ \phi$. Throughout this paper, $\phi, \psi$ and $\theta$ will denote these functors.

In Barnes [1], $A$ is an augmented algebra over a commutative ring $\mathfrak{K}$ and $C$ is the quotient $A / / B$ of $A$ by a normal augmented subalgebra. $A, B$ and $A / / B$ are all assumed to be projective as $\mathfrak{K}$-modules. The functor $\phi$ is given for the left $A$-module

Received by the editors April 30, 2001.

2000 Mathematics Subject Classification. Primary 18G40, 16W30; Secondary 16E40.

Key words and phrases. Spectral sequence, crossed product, comodule algebra, Hopf Galois extension.

This work was done while the author was an Honorary Associate of the School of Mathematics and Statistics, University of Sydney. 
$X$ by

$$
\phi X=X^{B}=\{x \in X \mid b x=\epsilon(b) x \text { for all } b \in B\} .
$$

For a $C$-module $Y, \psi Y=Y^{C}$ and we have $\psi \phi X=X^{A}$ for any $A$-module $X$. In this context, $\phi$ has a left adjoint $j: \mathcal{C} \rightarrow \mathcal{A}$, and use is made of the counit $\pi=j \phi: \mathcal{A} \rightarrow \mathcal{A}$ of the adjunction. Note that $\phi j=\mathrm{id}: \mathcal{C} \rightarrow \mathcal{C}$. We shall refer to this as the HS context.

In Guichardet's paper, $A$ is the crossed product $G \times{ }_{\alpha} B$, where $B$ is an algebra over the field $\mathfrak{K}, C$ is the group algebra $\mathfrak{K} G$ and the functor $\phi$ is given for the left $A^{e}$-module $X$ by

$$
\phi X=X^{B}=\{x \in X \mid b x=x b \text { for all } b \in B\}
$$

with the action of $G$ given by $g x=(g, 1) x\left(g^{-1}, 1\right)$ for $g \in G$ and $x \in X^{B}$. For the $\mathfrak{K} G$-module $Y$,

$$
\psi Y=Y^{G}=\{y \in Y \mid g y=y \text { for all } g \in G\} .
$$

In this context, $\phi$ does not have a left adjoint $j$. The assumption that $\mathfrak{K}$ is a field can be weakened if in some places we replace "injective" by "relatively injective". We require that $\mathfrak{K}$ is a commutative ring and that $B$ is $\mathfrak{K}$-projective. We shall refer to this as the $\mathrm{G}$ context.

In Stefan [5, $C$ is a Hopf algebra over the field $\mathfrak{K}, A$ is a $C$-comodule algebra and $B$ is the subalgebra of coinvariants. Thus we have an algebra morphism $\Delta_{A}$ : $A \rightarrow A \otimes C$ making $A$ a right $C$-comodule, and

$$
B=A^{\mathrm{co} C}=\left\{a \in A \mid \Delta_{A}(a)=a \otimes 1\right\} .
$$

Stefan refers to this situation as "the extension $A / B$ ". It is assumed to be $C$-Galois, which we explain in section 2 below. As in the $\mathrm{G}$ context, for the $A$-bimodule $X$, we set $\phi X=X^{B}$. This is made into a right $C$-module using the action defined by Stefan [5, Proposition 2.3] and explained following Lemma 2.1 below. For the $C$-module $Y$, we put $\psi Y=Y^{C}$. Again, we weaken the assumption that $\mathfrak{K}$ is a field. We require that $\mathfrak{K}$ is a commutative ring and that $A, B$ and $C$ are $\mathfrak{K}$-projective. We refer to this as the $\mathrm{S}$ context. It generalises the $\mathrm{G}$ context since the crossed product algebra $A=G \times{ }_{\alpha} B$ becomes a $C$-comodule algebra if we set $\Delta_{A}(g, b)=(g, b) \otimes g$ for $g \in G$ and $b \in B$. If in the HS (Hochschild-Serre) context, $A$ is a Hopf algebra, we may regard it as a $C$-comodule algebra with the comodule structure given by the comultiplication of $A$ followed by the natural homomorphism $A \otimes A \rightarrow A \otimes A / / B$. A left $A$-module $X$ may be regarded as a bimodule by setting $x a=\epsilon(a) x$ for $a \in A$ and $x \in X$, where $\epsilon$ is the augmentation. This does not change $\phi X=X^{B}$, now defined as $\{x \in X \mid b x=x b$ for all $b \in B\}$, nor does it change the $R^{q} \phi(X)$, although it does change the injective modules used for their calculation.

\section{Preliminaries}

We follow the notation for comodule algebras used in Schneider [4, with the exception that we denote the Hopf algebra by $C$, reserving the symbol $H$ for cohomology. Thus we have the comodule structure map, $\Delta_{A}: A \rightarrow A \otimes C$, and express the image of an element $a \in A$ by $\Delta_{A}(a)=\sum a_{0} \otimes a_{1}$. The comultiplication $\Delta_{C}: C \rightarrow C \otimes C$ is written $\Delta_{C}(c)=\sum c_{1} \otimes c_{2}$. The augmentation of $C$ is denoted by $\epsilon$ and the antipode by $S$. The canonical map can : $A \otimes_{B} A \rightarrow A \otimes C$ is defined by $\operatorname{can}\left(a \otimes_{B} a^{\prime}\right)=\sum a a_{0}^{\prime} \otimes a_{1}^{\prime}$. That $A / B$ is a Hopf Galois extension means that can is invertible, which we always assume. Thus for $c \in C$, there exist elements 
$r_{i}(c), l_{i}(c) \in A$, not uniquely determined, such that can $^{-1}(1 \otimes c)=\sum r_{i}(c) \otimes_{B} l_{i}(c)$, which is unique. We shall need the identities proved in Schneider [4, Remark 3.4(2)]. Throughout his paper, Schneider assumes $\mathfrak{K}$ to be a field, but his proof of the identities makes no use of that assumption. For the convenience of the reader, we list the identities here.

Lemma 2.1. For all $a \in A, b \in B$ and $c, c^{\prime} \in C$, the following identities hold:

(a) $\sum b r_{i}(c) \otimes_{B} l_{i}(c)=\sum r_{i}(c) \otimes_{B} l_{i}(c) b$,

(b) $\sum a_{0} r_{i}\left(a_{1}\right) \otimes_{B} l_{i}\left(a_{1}\right)=1 \otimes_{B} a$,

(c) $\sum r_{i}(c) l_{i}(c)=\epsilon(c)$,

(d) $\sum r_{i}(c) \otimes_{B} l_{i}(c)_{0} \otimes l_{i}(c)_{1}=\sum r_{i}\left(c_{1}\right) \otimes_{B} l_{i}\left(c_{1}\right) \otimes c_{2}$,

(e) $\sum r_{i}(c)_{0} \otimes_{B} l_{i}(c) \otimes r_{i}(c)_{1}=\sum r_{i}\left(c_{2}\right) \otimes_{B} l_{i}\left(c_{2}\right) \otimes S\left(c_{1}\right)$,

(f) $\sum r_{i}\left(c c^{\prime}\right) \otimes_{B} l_{i}\left(c c^{\prime}\right)=\sum r_{i}\left(c^{\prime}\right) r_{j}(c) \otimes_{B} l_{j}(c) l_{i}\left(c^{\prime}\right)$,

(g) $\sum r_{i}\left(c_{1}\right) \otimes_{B} l_{i}\left(c_{1}\right) r_{j}\left(c_{2}\right) \otimes_{B} l_{j}\left(c_{2}\right)=\sum r_{i}(c) \otimes_{B} 1 \otimes_{B} l_{i}(c)$.

Following Stefan [5. Proposition 2.3], we use the above relations to define a right $C$-module structure on $X^{B}$ for any left $A^{e}$-module $X$. For $x \in X^{B}$ and $c \in C$ we put $x \cdot c=\sum r_{i}(c) x l_{i}(c)$. This is well defined since $\sum r_{i}(c) \otimes_{B} l_{i}(c)$ is a welldefined element of $A \otimes_{B} A$ and $b x=x b$ for all $b \in B$. From 2.1(a), it follows that $x \cdot c \in X^{B}$. If a left action of $C$ on $X^{B}$ is preferred, one may be defined by setting $c \cdot x=x \cdot(S c)$. The assertion of Lemma 2.2 below holds for this left action provided that the antipode $S$ is bijective.

Lemma 2.2. For an $A^{e}$-module $X,\left(X^{B}\right)^{C}=X^{A}$.

Proof. For $x \in X^{A}$ and $c \in C$, we have

$$
x \cdot c=\sum r_{i}(c) x l_{i}(c)=\sum r_{i}(c) l_{i}(c) x=\epsilon(c) x
$$

by 2.1(c). Thus $x \in\left(X^{B}\right)^{C}$. Conversely, if $x \in\left(X^{B}\right)^{C}$, then by 2.1(b),

$$
x a=1 x a=\sum a_{0} r_{i}\left(a_{1}\right) x l_{i}\left(a_{1}\right)=\sum a_{0}\left(x \cdot a_{1}\right)=\sum a_{0} \epsilon\left(a_{1}\right) x=a x
$$

for all $a \in A$. Thus $x \in X^{A}$.

For the crossed product algebra $A=G \times{ }_{\alpha} B$, the canonical map is given by

$$
\operatorname{can}\left((g, b) \otimes_{B}\left(g^{\prime}, b^{\prime}\right)\right)=(g, b)\left(g^{\prime}, b^{\prime}\right) \otimes g^{\prime}=\left(g g^{\prime}, \alpha_{g^{\prime}}^{-1}(b) b^{\prime}\right) \otimes g^{\prime} .
$$

In particular, $\operatorname{can}\left(\left(g^{-1}, 1\right) \otimes_{B}(g, 1)\right)=(1,1) \otimes g$; so we can take $r(g)=\left(g^{-1}, 1\right)$ and $l(g)=(g, 1)$. The right action of $C=\mathfrak{K} G$ on $X^{B}$ becomes $x \cdot g=\left(g^{-1}, 1\right) x(g, 1)$ for $x \in X$ and $g \in G$. Converting this to a left action gives $g \cdot x=(g, 1) x\left(g^{-1}, 1\right)$, which is the action used in the Guichardet paper [3].

Note that, in the $\mathrm{G}$ context, $A^{e}=A \otimes A^{\mathrm{op}}$ is free as a right $B^{e}$-module. In the $\mathrm{S}$ context, we assume that $A$ is flat as left and right $B$-module. It then follows that $A^{e}$ is flat as right $B^{e}$-module. In the HS context, we assume that $A$ is at least projective as right $B$-module. At some points in [1], the stronger assumption that the module quotient $A / B$ is projective as right $B$-module is used. In all the contexts, by Barnes [1. Lemma I.4.3], every injective left $A$ - or $A^{e}$-module is injective as $B$ or $B^{e}$-module. Further, every injective of $\mathcal{A}$ or $\mathcal{C}$ is injective as $\mathfrak{K}$-module.

For any $\mathfrak{K}$-module $X$, the $A$-module coinduced from $X$ is the module $X^{*}=$ $\operatorname{Hom}_{\mathfrak{K}}(A, X)$ with the action $(a f)\left(a^{\prime}\right)=f\left(a^{\prime} a\right)$ for $f \in X^{*}$ and $a, a^{\prime} \in A$. (For the coinduced right module, the action is given by $(f a)\left(a^{\prime}\right)=f\left(a a^{\prime}\right)$.) If $X$ is itself a left $A$-module, then the map $\sigma: X \rightarrow X^{*}$ defined by $(\sigma x)(a)=a x$ for $x \in X$ and 
$a \in A$ is a $\mathfrak{K}$-split $A$-module monomorphism. $X^{*}$ is a relatively injective left $A$ module. (See for example, Barnes [1, Lemma II.2.4, p. 20].) A module is relatively injective if and only if it is a direct summand of a coinduced module. By Barnes [1, Lemma II.3.9, p. 28], if $A$ is right $B$-projective, then every relatively injective left $A$-module is relatively injective as left $B$-module. Thus in the $\mathrm{G}$ context, every relatively injective $A^{e}$-module is relatively injective as $B^{e}$-module. In the $\mathrm{S}$ context, to get this conclusion, we must strengthen Stefan's assumption that $A$ is left and right $B$-flat to $A$ being left and right $B$-projective, although as the next lemma shows, this strengthening is unnecessary if, as in [5], it is assumed that $\mathfrak{K}$ is a field, since then, every module is $\mathfrak{K}$-injective.

Lemma 2.3. If $X$ is $\mathfrak{K}$-injective, then the coinduced module $X^{*}$ is injective.

Proof. Let $i: V \rightarrow W$ be a monomorphism and $\alpha: V \rightarrow X^{*}$ a homomorphism of $A$-modules.

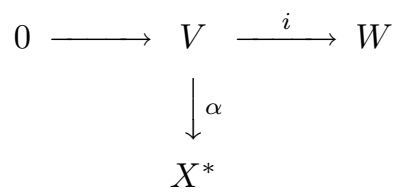

We want to construct a homomorphism $\beta: W \rightarrow X^{*}$ such that $\beta i=\alpha$. For $v \in V$, we have $\alpha v \in \operatorname{Hom}_{\mathfrak{K}}(A, X)$; so for $a \in A$, we have $(\alpha v)(a) \in X$. We may regard $\alpha$ as a function $A \times V \rightarrow X$, writing $\alpha(a, v)$ for $(\alpha v)(a)$. For $b \in A$, we have $\alpha(b v)=b(\alpha v)$. So $(\alpha(b v))(a)=(\alpha v)(a b)$; that is, $\alpha(a b, v)=\alpha(a, b v)$, and in particular $\alpha(a, v)=\alpha(1, a v)$. Putting $\bar{\alpha}(v)=(\alpha v)(1)$ creates the diagram

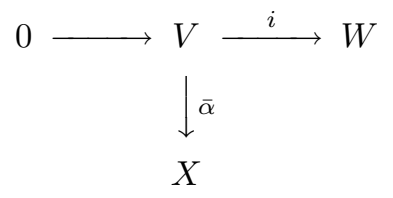

of $\mathfrak{K}$-modules. Since $X$ is $\mathfrak{K}$-injective, there exists a $\mathfrak{K}$-homomorphism $\bar{\beta}: W \rightarrow X$ such that $\bar{\beta} i=\bar{\alpha}$. Define $\beta: W \rightarrow X^{*}$ by $\beta(w)(a)=\bar{\beta}(a w)$. Then for $b \in A$, we have

$$
\beta(b w)(a)=\bar{\beta}(a b w)=(\beta w)(a b)=(b \beta(w))(a) ;
$$

so $\beta$ is an $A$-module homomorphism. We have

$$
(\beta i v)(a)=\bar{\beta}(a(i v))=\bar{\beta}(i(a v))=\bar{\alpha}(a v)=(\alpha v)(a)
$$

and $\beta i=\alpha$.

We have defined a right $C$-module action on $X^{B}$ for any left $A^{e}$-module $X$. We need another description of that action in the case where $X$ is a coinduced module.

Lemma 2.4. Let $V$ be a $\mathfrak{K}$-module and let $V^{*}=\operatorname{Hom}\left(A^{e}, V\right)$ be the coinduced $A^{e}$ module. Then $V^{* B}$ is isomorphic to the coinduced $C$-module $\operatorname{Hom}(C, \operatorname{Hom}(A, V))$.

Proof. The action of $a \otimes a^{\prime} \in A^{e}$ on $f \in V^{*}$ is given by

$$
\left(\left(a \otimes a^{\prime}\right) f\right)(x \otimes y)=f\left((x \otimes y)\left(a \otimes a^{\prime}\right)\right)=f\left(x a \otimes a^{\prime} y\right)
$$

for $x, y \in A$. Now

$$
\begin{aligned}
V^{* B} & =\left\{f \in V^{*} \mid(b \otimes 1) f=(1 \otimes b) f \text { for all } b \in B\right\} \\
& =\left\{f \in V^{*} \mid f(x b \otimes y)=f(x \otimes b y) \text { for all } b \in B \text { and } x, y \text { in } A\right\} .
\end{aligned}
$$


Thus $V^{* B}$ can be identified with $\operatorname{Hom}\left(A \otimes_{B} A, V\right)$, and so, using the canonical map, with $\operatorname{Hom}(A \otimes C, V)=\operatorname{Hom}(C, \operatorname{Hom}(A, V))$. We calculate the right $C$-module action on $\operatorname{Hom}(C, \operatorname{Hom}(A, V))$ induced by these identifications. For $f \in V^{* B}$ and $c \in C$, from the $A^{e}$-action on $V$, we get $f c=\sum\left(r_{i}(c) \otimes l_{i}(c)\right) f$. Thus

$$
(f c)(x \otimes y)=\sum f\left(x r_{i}(c) \otimes l_{i}(c) y\right) .
$$

For $f \in \operatorname{Hom}(C, \operatorname{Hom}(A, V))$ we have the corresponding $f^{\prime} \in \operatorname{Hom}(A \otimes C, V)$ given by $f^{\prime}(a \otimes c)=f(c)(a)$ and $f^{\prime \prime} \in \operatorname{Hom}\left(A \otimes_{B} A, V\right)$ given by

$$
f^{\prime \prime}\left(x \otimes_{B} y\right)=f^{\prime}\left(\operatorname{can}\left(x \otimes_{B} y\right)\right)=\sum f^{\prime}\left(x y_{0} \otimes y_{1}\right) .
$$

Thus,

$$
f(c)(a)=f^{\prime}(a \otimes c)=\sum f^{\prime \prime}\left(a r_{i}(c) \otimes_{B} l_{i}(c)\right) .
$$

So for $c^{\prime} \in C, f c^{\prime}$ is given by

$$
\begin{aligned}
\left(f c^{\prime}\right)(c)(a) & =\sum\left(f^{\prime \prime} c^{\prime}\right)\left(a r_{i}(c) \otimes_{B} l_{i}(c)\right) \\
& =\sum f^{\prime \prime}\left(a r_{i}(c) r_{j}\left(c^{\prime}\right) \otimes_{B} l_{j}\left(c^{\prime}\right) l_{i}(c)\right) \\
& =\sum f^{\prime \prime}\left(a r_{i}\left(c^{\prime} c\right) \otimes_{B} l_{i}\left(c^{\prime} c\right)\right) \quad \text { by } 2.1(\mathrm{f}) \\
& =f\left(c^{\prime} c\right)(a) .
\end{aligned}
$$

Thus the action $\left(f c^{\prime}\right)(c)=f\left(c^{\prime} c\right)$ is that of the coinduced right $C$-module.

The next result strengthens Stefan [5, Proposition 3.2]. The corresponding result in the HS context follows easily from the fact that every $A / / B$-module is an $A$ module and that $Q^{B}$ is a submodule of $Q$.

Lemma 2.5 . Let $Q$ be a relatively injective $A^{e}$-module. Then $Q^{B}$ is a relatively injective $C$-module. If $Q$ is injective, then $Q^{B}$ is injective.

Proof. $Q$ is a direct summand of some coinduced module $V^{*}=\operatorname{Hom}_{\mathfrak{K}}\left(A^{e}, V\right)$. So to prove $Q^{B}$ relatively injective, it is sufficient to show that $\operatorname{Hom}_{\mathfrak{K}}\left(A^{e}, V\right)^{B}$ is relatively injective. $\operatorname{But} \operatorname{Hom}(C, \operatorname{Hom}(A, V))$ is relatively injective; so by Lemma $2.4, V^{* B}$ is relatively injective. Thus $Q^{B}$ is relatively injective. If $Q$ is injective, we may take $V=Q$. Since $Q$ is $\mathfrak{K}$-injective, by Lemma $2.3, \operatorname{Hom}(A, Q)$ is also $\mathfrak{K}$-injective and $\operatorname{Hom}(C, \operatorname{Hom}(A, Q))$ is an injective $C$-module. Thus $Q^{B}$ is injective.

\section{Spectral sequence constructors generalised}

In Barnes [1, Chapter III] in the HS context, a spectral sequence constructor for $(\phi, \psi)$ was defined to be a functor $F$ from $\mathcal{A}$ to filtered cochain complexes in $\mathcal{D}$ such that

(1) $F$ is exact (in every filtration).

(2) $F$ is acyclic on injectives; that is, if $Q$ is injective, then $H^{n}(F Q)=0$ for $n>0$ and for all $p, H^{q}\left(\mathcal{F}^{p} Q /{ }^{\bullet} F^{p+1} Q\right)=0$ for $q>0$.

(3) $E_{1}^{\bullet 0} F$ is exact on $\mathcal{C}$.

(4) The inclusion $i: X^{B} \rightarrow X$ induces isomorphisms $E_{1}^{p 0} F\left(X^{B}\right) \rightarrow E_{1}^{p 0} F(X)$ for all $p$ and all $X \in \mathcal{A}$.

(5) $H^{0} F$ is naturally isomorphic to $\psi \phi$. 
Here, using the fact that $A / / B$-modules are $A$-modules, that is, using the adjoint $j$ to $\phi$, we construct a functor $\Gamma=E_{1}^{\bullet 0} F j$ from $\mathcal{C}$ to cochain complexes in $\mathcal{D}$. This cannot be done in the $\mathrm{G}$ or $\mathrm{S}$ contexts. So we must include the cochain complex functor as part of the structure in our definition of a constructor. If $F$ is a filtered cochain complex, we denote the component of total degree $n$ by ${ }^{n} F$, the $p^{\text {th }}$ filtration by $F^{p}$, the submodule of filtration degree $p$ and complementary degree $q$ by $F^{p q}$ and use similar notation for the terms of its spectral sequence $E(F)$. We always assume that ${ }^{n} F^{0}={ }^{n} F$ and that ${ }^{n} F^{n+1}=0$. The following definition generalises the one quoted above.

Definition 3.1. A spectral sequence constructor for the pair $(\phi, \psi)$ is a quadruple $(F, \Gamma, \eta, \gamma)$, where $F$ is a functor from $\mathcal{A}$ to filtered cochain complexes in $\mathcal{D}, \Gamma$ is a functor from $\mathcal{C}$ to cochain complexes in $\mathcal{D}, \eta$ is a natural isomorphism $E_{1}^{\bullet 0}(F) \rightarrow \Gamma \phi$ and $\gamma$ is a natural isomorphism $H^{0}(\Gamma) \rightarrow \psi$ such that

(1) $F$ is exact (in every filtration).

(2) $F$ is acyclic on injectives; that is, if $Q$ is injective, then $H^{n}(F Q)=0$ for $n>0$ and for all $p, H^{q}\left(\bullet^{\bullet} F^{p} Q /{ }^{\bullet} F^{p+1} Q\right)=0$ for $q>0$.

(3) $\Gamma$ is exact and acyclic on injectives.

From (1), it follows that $F^{p} / F^{p+r}$ is exact for all $p, r$. From $\gamma$ being a natural isomorphism and (3), it follows that we have a unique family of natural isomorphisms $\gamma^{p}: H^{p} \Gamma \rightarrow R^{p} \psi$, the right derived functors of $\psi$, commuting with connecting homomorphisms, and with $\gamma^{0}=\gamma$. We denote this family by $\gamma$. Furthermore,

$$
H^{0} F=E_{\infty}^{00} F=E_{2}^{00} F=H^{0} E_{1}^{\bullet 0} F=H^{0} \eta^{-1} \Gamma \phi
$$

and so

$$
\gamma H^{0}(\eta) H^{0} F=\gamma \phi=\theta ;
$$

that is, $\gamma H^{0}(\eta)$ is a natural isomorphism from $H^{0} F$ to $\theta$. It follows that $H^{n} F=R^{n} \theta$ for all $n$.

We now prove the results corresponding to Barnes [1, Theorem III.2.3, p. 42] and the lemmas leading up to that theorem, beginning with the analogue of [1. Theorem III.1.5, p. 38].

Lemma 3.2. Let $(F, \Gamma, \eta, \gamma)$ be a spectral sequence constructor. There exists a unique family of natural transformations $\eta^{p q}: E_{1}^{p q}(F) \rightarrow \Gamma^{p}\left(R^{q} \phi\right)$ commuting with connecting homomorphisms and with $\eta^{p 0}=\eta^{p}$. The $\eta^{p q}$ are natural isomorphisms, satisfy $\eta^{p+1, q} d_{1}^{p q}=(-1)^{q} \delta \eta^{p q}$, where $\delta$ is the differential of $\Gamma$, and induce natural isomorphisms

$$
H^{p}\left(\eta^{\bullet q}\right): E_{2}^{p q}(F) \rightarrow H^{p}\left(\Gamma\left(R^{q} \phi\right)\right)
$$

and

$$
\gamma H^{p}\left(\eta^{\bullet q}\right): E_{2}^{p q}(F) \rightarrow\left(R^{p} \psi\right)\left(R^{q} \phi\right) .
$$

Proof. $\Gamma$ is exact by assumption. So $\left\{\Gamma^{p}\left(R^{q} \phi\right) \mid q=0,1, \ldots\right\}$ is a connected sequence of functors, as is $\left\{E_{1}^{p q}(F)=H^{q}\left(F^{p} / F^{p+1}\right) \mid q=0,1, \ldots\right\}$. Both vanish for $q>0$ on injectives. By dimension-shifting, it follows that there exists a unique family of natural transformations $\eta^{p q}: E_{1}^{p q}(F) \rightarrow \Gamma^{p}\left(R^{q} \phi\right)$ extending the given transformation $\eta^{p}: E_{1}^{p 0}(F) \rightarrow \Gamma^{p} \phi$. Since $\eta^{p 0}$ is a natural isomorphism, all the $\eta^{p q}$ are natural isomorphisms. The argument of [1, pp. 38, 39] applies unchanged to give the result. 
Definition 3.3. A natural transformation $\xi:(F, \Gamma, \eta, \gamma) \rightarrow\left(F^{\prime}, \Gamma^{\prime}, \eta^{\prime} \gamma^{\prime}\right)$ of constructors is a pair $\xi_{F}: F \rightarrow F^{\prime}$ and $\xi_{\Gamma} \Gamma \rightarrow \Gamma^{\prime}$ of natural transformations such that the diagrams

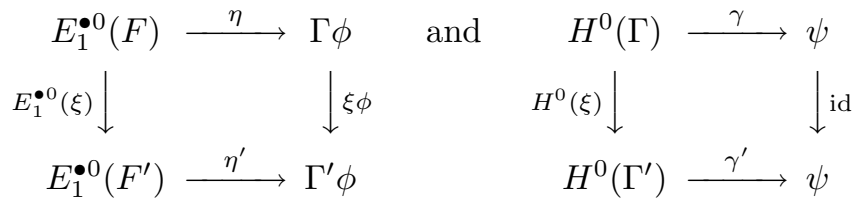

commute.

We shall omit the subscripts from $\xi_{F}$ and $\xi_{\Gamma}$. Our next lemma is easier than [1, II.2.2] in that the transformation $\xi: \Gamma \rightarrow \Gamma^{\prime}$ is given instead of having to be constructed.

Lemma 3.4. Let $\xi:(F, \Gamma, \eta, \gamma) \rightarrow\left(F^{\prime}, \Gamma^{\prime}, \eta^{\prime}, \gamma^{\prime}\right)$ be a natural transformation of constructors. Then the diagram

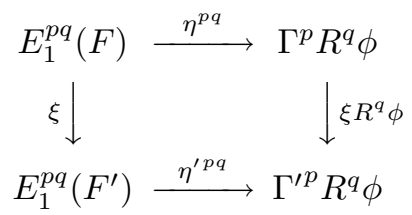

commutes for all $p$ and $q$.

Proof. $\xi R^{\bullet} \phi$ is a natural transformation of connected sequences of functors. Since in dimension $q=0$, we have $\xi R^{0} \phi=\eta^{\prime p 0} \xi\left(\eta^{p 0}\right)^{-1}$, by dimension-shifting, we have $\xi R^{q} \phi=\eta^{\prime p q} \xi\left(\eta^{p q}\right)^{-1}$ for all $q$.

Theorem 3.5. Let $\xi:(F, \Gamma, \eta, \gamma) \rightarrow\left(F^{\prime}, \Gamma^{\prime}, \eta^{\prime}, \gamma^{\prime}\right)$ be a natural transformation of constructors. Then $\xi$ induces a natural isomorphism $\xi_{E}: E(F) \rightarrow E\left(F^{\prime}\right)$ of their spectral sequences, that is, $\xi_{r}^{p q}: E_{r}^{p q}(F) \rightarrow E_{r}^{p q}\left(F^{\prime}\right)$ is a natural isomorphism for all $r \geq 2$ and all $p, q$.

Proof. By assumption, $\gamma: H^{0}(\Gamma) \rightarrow \psi$ and $\gamma^{\prime}: H^{0}\left(\Gamma^{\prime}\right) \rightarrow \psi$ are natural isomorphisms, and $\gamma^{\prime} H^{0}(\xi)=\gamma$. Therefore $H^{0}(\xi)=\left(\gamma^{\prime}\right)^{-1} \gamma$ is a natural isomorphism. By dimension-shifting, it follows that $H^{p}(\xi): H^{p} \Gamma \rightarrow H^{p} \Gamma^{\prime}$ is a natural isomorphism for all $p$. The diagram

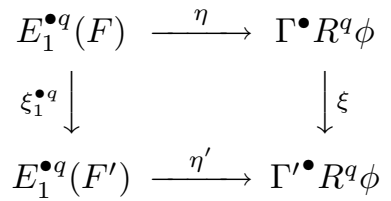

is, up to sign, a commutative diagram of cochain complexes. (If $q$ is odd, $\eta$ and $\eta^{\prime}$ anticommute with the differentials.) Taking $H^{p}$ of this, we get the commutative diagram

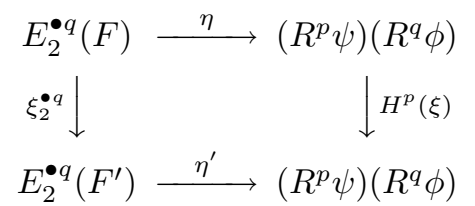

in which $\eta, \eta^{\prime}$ and $H^{p}(\xi)$ are natural isomorphisms. It follows that $\xi_{2}^{p q}$ is a natural isomorphism and so, that $\xi_{r}^{p q}$ is a natural isomorphism for all $r \geq 2$. 


\section{Guichardet's First CONStRuctor}

For the $A^{e}$-module $X$, Guichardet defines the double complex

$$
K^{p q}(X)=\left(\mathcal{F}\left(G^{p+1}, \operatorname{Hom}\left(\otimes^{q+1} A^{e}, X\right)\right)^{B}\right)^{G}
$$

with appropriately defined differential, where $\mathcal{F}(U, V)$ denotes the set of functions from the set $U$ to the set $V$. Following Guichardet [3], we set $I^{n}(X)=$ $\operatorname{Hom}\left(\otimes^{n+1} A^{e}, X\right)$ with differential

$$
\begin{aligned}
& d f\left(a_{0} \otimes a_{0}^{\prime}, \ldots, a_{n+1} \otimes a_{n+1}^{\prime}\right) \\
& =a_{0} f\left(a_{1} \otimes a_{1}^{\prime}, \ldots, a_{n+1} \otimes a_{n+1}^{\prime}\right) a_{0}^{\prime} \\
& \quad+\sum_{i=0}^{n}(-1)^{i+1} f\left(a_{0} \otimes a_{0}^{\prime}, \ldots, a_{i} a_{i+1} \otimes a_{i+1}^{\prime} a_{i}^{\prime}, \ldots, a_{n+1} \otimes a_{n+1}^{\prime}\right),
\end{aligned}
$$

which gives a relatively injective resolution $I^{\bullet}(X)$ of $X$ in $\mathcal{A}$. Also following Guichardet, we put $P_{n}=\otimes^{n+1} \mathfrak{K} G$ with action $g\left(g_{0} \otimes \ldots \otimes g_{n}\right)=g g_{0} \otimes \ldots \otimes g g_{n}$ and set $d\left(g_{0} \otimes \ldots \otimes g_{n}\right)=\sum_{i=0}^{n}(-1)^{i}\left(g_{0} \otimes \ldots \widehat{g}_{i} \ldots \otimes g_{n}\right)$ and $\epsilon\left(g_{0}\right)=1$. This makes $P \bullet$ a free resolution of $\mathfrak{K}$ in $\mathcal{C}$. We then have

$$
K^{p q}(X)=\operatorname{Hom}_{\mathfrak{K} G}\left(P_{p}, I^{q}(X)^{B}\right) .
$$

Expressed in this way, it is the Grothendiek repeated (relatively) injective resolution construction for the spectral sequence of a composite functor discussed in Barnes [1, Chapter VII], with $\operatorname{Hom}_{\mathfrak{K}}\left(P_{\bullet}\right.$, ) used as the relatively injective resolution functor on $\mathcal{C}$. For any relatively injective resolution functor $I^{\bullet}$ and any projective resolution $P_{\bullet}$, setting $K^{p q}(X)=\operatorname{Hom}_{\mathfrak{K} G}\left(P_{p}, I^{q}(X)^{B}\right)$ gives a constructor $(K, \Gamma, \eta, \gamma)$ with $\Gamma=\operatorname{Hom}_{\mathfrak{K} G}\left(P_{p},\right), \eta=$ id and $\gamma=$ id. The spectral sequence constructed is independent (from the $E_{2}$-level onward) of the choice of $I^{\bullet}$ and of $P_{\bullet}$.

This constructor may also be regarded as an adaptation of the Cartan and Eilenberg pair of resolutions constructor discussed in Barnes [1, Chapter VI]. Since $C$ modules are not $A^{e}$-modules, we cannot use $\operatorname{Hom}_{A^{e}}\left(P_{p}, I^{q}\right)$ as in the HS context, but use instead $\operatorname{Hom}_{C}\left(P_{p},\left(I^{q}\right)^{B}\right)$ which, in the HS context, is essentially the same.

Stefan in [5] establishes the conditions for the Grothendiek composite functor spectral sequence. To obtain a spectral sequence constructor, we have merely to assign functorially the resolutions used in the construction. If we assume that $A$ is left and right $B$-projective or if we assume that $\mathfrak{K}$ is a field, then we can use the $I^{n}$ defined as above and any right $C$-module projective resolution $P$ • of $\mathfrak{K}$.

\section{Guichardet's SECOND CONSTRUCtor}

For his second construction, Guichardet defines a filtration on the normalised standard complex ${ }^{\bullet} N(A, X)$ where ${ }^{n} N(A, X)$ is the subspace of $\operatorname{Hom}_{\mathfrak{K}}\left(\otimes^{n} A, X\right)$ of functions $f$ for which $f\left(a_{1}, \ldots, a_{n}\right)=0$ if any of the $a_{i}$ is in $\mathfrak{K} 1$, and

$$
\begin{aligned}
& d f\left(a_{1}, \ldots, a_{n+1}\right) \\
& =a_{1} f\left(a_{2}, \ldots, a_{n+1}\right)+\sum_{i=1}^{n}(-1)^{i} f\left(a_{1}, \ldots, a_{i} a_{i+1}, \ldots, a_{n+1}\right) \\
& +(-1)^{n+1} f\left(a_{1}, \ldots, a_{n}\right) a_{n+1} .
\end{aligned}
$$


The filtration on this complex is given by defining ${ }^{n} N^{0}={ }^{n} N$ and ${ }^{n} N^{p}$ for $1 \leq p \leq n$ to be the subset of those functions $f$ satisfying

$$
\begin{aligned}
& f\left(a_{1}, \ldots, a_{q}, g_{1} b_{1}, \ldots, g_{p} b_{p}\right) \\
& \quad=f\left(a_{1}, \ldots, a_{q}, g_{1}, \ldots, g_{p}\right) \alpha_{g_{2} g_{3} \ldots g_{p}}^{-1}\left(b_{1}\right) \alpha_{g_{3} \ldots g_{p}}^{-1}\left(b_{2}\right) \ldots \alpha_{g_{p}}^{-1}\left(b_{p-1}\right) b_{p} .
\end{aligned}
$$

Guichardet takes for $\Gamma$ the normalised standard complex and constructs a natural transformation from $E_{1}^{\bullet 0}(N)$ to $\Gamma \phi$ which, in [3, Lemme 3.11], he shows is a natural isomorphism. The conditions for a spectral sequence constructor are clearly satisfied. The purpose of this section is to generalise this to the $\mathrm{S}$ context.

To use the normalised standard complex in the $\mathrm{S}$ context, we must impose a further condition on the algebra $A$. The theory of the normalised standard complex (Cartan and Eilenberg [2] p. 176]) requires that the quotient $\bar{A}=A / \mathfrak{K} 1$ be projective as $\mathfrak{K}$-module. We assume this in this section. Equivalently, we assume that there exists a $\mathfrak{K}$-linear map $\epsilon: A \rightarrow \mathfrak{K}$ such that $\epsilon(k 1)=k$. This condition always holds if $\mathfrak{K}$ is a field or if, as in the HS context, $A$ is an augmented algebra.

An equivalent definition of ${ }^{n} N^{p}$, also given by Guichardet, is meaningful in the $\mathrm{S}$ context. So we use it here but with sides reversed because of our use of right comodule algebras and right $C$-modules. For $p \geq 1$, we define ${ }^{n} N^{p}$ to be the subset of ${ }^{n} N(A, X)$ of those functions satisfying

$$
f\left(b a_{1}, \ldots, a_{n-1}, a_{n}\right)=b f\left(a_{1}, \ldots, a_{n}\right)
$$

and

$$
f\left(a_{1}, \ldots, a_{i-1} b, a_{i}, \ldots, a_{n}\right)=f\left(a_{1}, \ldots, a_{i-1}, b a_{i}, \ldots, a_{n}\right) \text { for } i=2, \ldots, p
$$

for all $a_{1}, \ldots, a_{n} \in A$ and $b \in B$.

The normalised standard complex $\bullet N(C, Y)$ for a right $C$-module $Y$ is that obtained by treating $Y$ as a bimodule with left action $c \cdot y=\epsilon(c) y$. Thus, ${ }^{n} N(C, Y)$ is the subspace of $\operatorname{Hom}_{\mathfrak{K}}\left(\otimes^{n} C, Y\right)$ of functions $f$ for which $f\left(c_{1}, \ldots, c_{n}\right)=0$ if any of the $c_{i}$ is in $\mathfrak{K} 1$, with the differential

$$
\begin{array}{rl}
d f\left(c_{1}, \ldots, c_{n+1}\right)=\epsilon\left(c_{1}\right) f\left(c_{2}, \ldots, c_{n+1}\right)+\sum_{i=1}^{n}(-1)^{i} & f\left(c_{1}, \ldots, c_{i} c_{i+1}, \ldots, c_{n+1}\right) \\
& +(-1)^{n+1} f\left(c_{1}, \ldots, c_{n}\right) c_{n+1} .
\end{array}
$$

We put $T^{p q}={ }^{p} N\left(C,{ }^{q} N(B, X)\right)$ and write $N^{p q}$ for ${ }^{p+q} N^{p}$. Note that, although $N(B, X)$ is not, in general, a $C$-module, this does define $\mathfrak{K}$-modules $T^{p q}$.

For $f \in N^{p q}(A, X)$, we put

$$
\begin{aligned}
& \Psi^{p q}(f)\left(c_{1}, \ldots, c_{p}\right)\left(b_{1}, \ldots, b_{q}\right) \\
& \quad=\sum r_{i_{p}}\left(c_{p}\right) \ldots r_{i_{1}}\left(c_{1}\right) f\left(l_{i_{1}}\left(c_{1}\right), \ldots, l_{i_{p}}\left(c_{p}\right), b_{1}, \ldots, b_{q}\right) .
\end{aligned}
$$

That $\Psi^{p q}: N^{p q} \rightarrow T^{p q}$ is a well-defined $\mathfrak{K}$-linear map follows from the next lemma. We shorten the notation by writing $\vec{a}$ for a string $a_{1}, \ldots, a_{q}$ of elements of $A$ of any length. We further shorten notation by omitting unnecesary subscripts from the $r_{i}(c), l_{i}(c)$.

Lemma 5.3. If $f \in N^{p q}(A, X)$, then for $j=1, \ldots, p$, and all $\vec{a} \in A, b \in B$ and $c, c_{1}, \ldots, c_{p} \in C$,

(1) $\sum r\left(c_{j}\right) \ldots r\left(c_{1}\right) f\left(l\left(c_{1}\right), \ldots, l\left(c_{j}\right), \vec{a}\right)$ is independent of the choice of the $r\left(c_{j}\right)$ and $l\left(c_{j}\right)$. 
(2) $\sum b r\left(c_{j}\right) r\left(c_{j-1}\right) \ldots r\left(c_{1}\right) f\left(l\left(c_{1}\right), \ldots, l\left(c_{j}\right), \vec{a}\right)$

$=\sum r\left(c_{j}\right) \ldots r\left(c_{1}\right) f\left(l\left(c_{1}\right), \ldots, l\left(c_{j}\right) b, \vec{a}\right)$.

(3) $\sum r(c) r\left(c_{j}\right) \ldots r\left(c_{1}\right) f\left(l\left(c_{1}\right), \ldots, l\left(c_{j}\right) l(c), \vec{a}\right)$

$=\sum r\left(c_{j} c\right) \ldots r\left(c_{1}\right) f\left(l\left(c_{1}\right), \ldots, l\left(c_{j} c\right), \vec{a}\right)$.

Proof. For $a, a^{\prime} \in A$, we put

$$
g_{j}\left(a \otimes_{B} a^{\prime}\right)=\sum \operatorname{ar}\left(c_{j-1}\right) \ldots r\left(c_{1}\right) f\left(l\left(c_{1}\right), \ldots, l\left(c_{j-1}\right), a^{\prime}, \vec{a}\right) .
$$

By the condition (5.1), $g_{1}$ is well defined. Thus (1) holds for $j=1$. Also, by putting

$$
a \otimes_{B} a^{\prime}=\sum b r\left(c_{1}\right) \otimes_{B} l\left(c_{1}\right)=\sum r\left(c_{1}\right) \otimes_{B} l\left(c_{1}\right) b,
$$

by Lemma 2.1(a), we see that (2) holds for $j=1$. We use induction over $j$.

For $1<j \leq p$, we have

$$
\begin{aligned}
\sum a b r\left(c_{j-1}\right) \ldots r\left(c_{1}\right) f\left(l\left(c_{1}\right), \ldots, l\left(c_{j-1}\right), a^{\prime}, \vec{a}\right) \\
\quad=\sum a r\left(c_{j-1}\right) \ldots r\left(c_{1}\right) f\left(l\left(c_{1}\right), \ldots, l\left(c_{j-1}\right) b, a^{\prime}, \vec{a}\right) \\
=\sum a r\left(c_{j-1}\right) \ldots r\left(c_{1}\right) f\left(l\left(c_{1}\right), \ldots, l\left(c_{j-1}\right), b a^{\prime}, \vec{a}\right)
\end{aligned}
$$

by the induction hypothesis that (2) holds for $j-1$ and condition (5.2). Thus $g_{j}$ is well defined. Putting $a \otimes_{B} a^{\prime}=\sum r\left(c_{j}\right) \otimes_{B} l\left(c_{j}\right)$ gives the assertion (1). Putting $a \otimes_{B} a^{\prime}=\sum b r\left(c_{j}\right) \otimes_{B} l\left(c_{j}\right)$ and using Lemma 2.1(a) gives (2). Putting $a \otimes_{B} a^{\prime}=\sum r(c) r\left(c_{j}\right) \otimes_{B} l\left(c_{j}\right) l(c)$ and using Lemma 2.1(f) gives (3).

Lemma 5.4. $\Psi$ defines a natural cochain map $\Psi_{0}^{p \bullet}: E_{0}^{p \bullet} \rightarrow T^{p \bullet}$.

Proof. If $f \in{ }^{n} N^{p+1}$, then

$$
\begin{aligned}
\Psi^{p q}(f)\left(c_{1}, \ldots, c_{p}\right)\left(b_{1}, \ldots, b_{q}\right) & =\sum r\left(c_{p}\right) \ldots r\left(c_{1}\right) f\left(l\left(c_{1}\right), \ldots, l\left(c_{p}\right) b_{1}, 1, b_{2}, \ldots, b_{q}\right) \\
& =0 .
\end{aligned}
$$

Since ${ }^{n} E_{0}^{p}={ }^{n} N^{p} /{ }^{n} N^{p+1}, \Psi^{p q}$ defines a $\mathfrak{K}$-linear map $\Psi_{0}^{p q}: E_{0}^{p q} \rightarrow T^{p q}$. Consider the expression for $\sum\left(d_{A} f\right)\left(l\left(c_{1}\right), \ldots, l\left(c_{p}\right), b_{1}, \ldots, b_{q+1}\right)$. For those terms in which the string of $l\left(c_{i}\right)$ 's is reduced in length, we get $b_{1}$ in the $p^{\text {th }}$ place; so those terms are 0 . Thus,

$$
\begin{aligned}
\left(\Psi^{p, q+1}\right. & \left.d_{A} f\right)\left(c_{1}, \ldots, c_{p}\right)\left(b_{1}, \ldots, b_{q+1}\right) \\
= & (-1)^{p} \sum r\left(c_{p}\right) \ldots r\left(c_{1}\right) f\left(l\left(c_{1}\right), \ldots, l\left(c_{p}\right) b_{1}, \ldots, b_{q+1}\right) \\
& \quad+\sum(-1)^{p+i} r\left(c_{p}\right) \ldots f\left(\ldots,\left(b_{i} b_{i+1}\right), \ldots\right) \\
& \quad+(-1)^{p+q+1} \sum r\left(c_{p}\right) \ldots f\left(l\left(c_{1}\right), \ldots, b_{q}\right) b_{q+1} \\
= & (-1)^{p} d_{B}\left(\left(\Psi^{p q} f\right)\left(c_{1}, \ldots, c_{p}\right)\right)\left(b_{1}, \ldots, b_{q+1}\right)
\end{aligned}
$$

by applying Lemma $5.3(2)$ to the first term. The result follows, the naturality being obvious.

We are trying to construct a spectral sequence constructor using $N_{A}=N(A, \quad)$ with the Guichardet filtration as the filtered complex functor. Clearly, we can set $\Gamma=N(C, \quad)$ and $\gamma=\mathrm{id}: H^{0}(C, \quad) \rightarrow \psi$. We still need a natural isomorphism $\eta: E_{1}^{\bullet 0}\left(N_{A}\right) \rightarrow \Gamma \phi$. Applying $H^{q}$ to the natural cochain map $\Psi_{0}^{p \bullet}$ gives a natural map $\eta^{p q}: E_{1}^{p q} \rightarrow{ }^{p} N\left(C, H^{q}(B, \quad)\right)=\Gamma^{p}\left(H^{q}(B, \quad)\right)$. We must first show that $\eta^{\bullet 0}$ is an isomorphism of cochain complexes. 
Lemma 5.5. $\eta^{\bullet 0}: E_{1}^{\bullet 0}(X) \rightarrow \Gamma^{\bullet} X^{B}$ is a map of cochain complexes.

Proof. An element of $E_{1}^{p 0}(X)$ is represented by a function $f \in{ }^{p} N^{p}(A, X)$ such that $d f \in{ }^{p+1} N^{p+1}$. For $f \in{ }^{p} N^{p}$, every term $t$ in $d f$ satisfies (5.1) and (5.2) for all $i$ except the term $t\left(a_{1}, \ldots, a_{p+1}\right)=f\left(a_{1}, \ldots, a_{p}\right) a_{p+1}$ for which (5.2) may fail for $i=p+1$. Thus the requirement that $d f \in{ }^{p+1} N^{p+1}$ imposes the one extra condition that $f\left(a_{1}, \ldots, a_{p} b\right) a_{p+1}=f\left(a_{1}, \ldots, a_{p}\right) b a_{p+1}$, that is, $f\left(a_{1}, \ldots, a_{p} b\right)=f\left(a_{1}, \ldots, a_{p}\right) b$. For such an $f$, we have, writing $\vec{c}$ for $c_{1}, \ldots, c_{p+1}$,

$$
\begin{aligned}
\left(\Psi^{p+1,0} d_{A} f\right)(\vec{c})= & \sum r\left(c_{p+1}\right) \ldots r\left(c_{1}\right) d f\left(l\left(c_{1}\right), \ldots, l\left(c_{p+1}\right)\right) \\
= & \sum r\left(c_{p+1}\right) \ldots r\left(c_{1}\right) l\left(c_{1}\right) f\left(l\left(c_{2}\right), \ldots, l\left(c_{p+1}\right)\right) \\
& +\sum(-1)^{i} r\left(c_{p+1}\right) \ldots r\left(c_{1}\right) f\left(l\left(c_{1}\right), \ldots,\left(c_{i}\right) l\left(c_{i+1}\right), \ldots, l\left(c_{p+1}\right)\right) \\
& +\sum(-1)^{p+1} r\left(c_{p+1}\right) \ldots r\left(c_{1}\right) f\left(l\left(c_{1}\right), \ldots, l\left(c_{p}\right)\right) l\left(c_{p+1}\right) .
\end{aligned}
$$

By Lemma 2.1(c),

$$
\begin{aligned}
\sum r\left(c_{p+1}\right) & \ldots r\left(c_{1}\right) l\left(c_{1}\right) f\left(l\left(c_{2}\right), \ldots, l\left(c_{p+1}\right)\right) \\
= & \epsilon\left(c_{1}\right) \sum r\left(c_{p+1}\right) \ldots r\left(c_{2}\right) f\left(l\left(c_{2}\right), \ldots, l\left(c_{p+1}\right)\right) .
\end{aligned}
$$

By Lemma 5.3(3),

$$
\begin{aligned}
& \sum(-1)^{i} r\left(c_{p+1}\right) \ldots r\left(c_{1}\right) f\left(l\left(c_{1}\right), \ldots,\left(c_{i}\right) l\left(c_{i+1}\right), \ldots, l\left(c_{p+1}\right)\right) \\
& \quad=\sum(-1)^{i} r\left(c_{p+1}\right) \ldots r\left(c_{i} c_{i+1}\right) \ldots r\left(c_{1}\right) f\left(l\left(c_{1}\right), \ldots,\left(c_{i} c_{i+1}\right), \ldots, l\left(c_{p+1}\right)\right) .
\end{aligned}
$$

Also,

$$
\begin{aligned}
\sum r\left(c_{p+1}\right) & \ldots r\left(c_{1}\right) f\left(l\left(c_{1}\right), \ldots, l\left(c_{p}\right)\right) l\left(c_{p+1}\right) \\
= & \left(\sum r\left(c_{p}\right) \ldots r\left(c_{1}\right) f\left(l\left(c_{1}\right), \ldots, l\left(c_{p}\right)\right)\right) \cdot c_{p+1} .
\end{aligned}
$$

Thus $\Psi^{p+1,0} d_{A} f=d_{C} \Psi^{p 0} f$ and the result follows.

For $g \in{ }^{p} N\left(C, X^{B}\right)$, we define $\Phi g \in{ }^{p} N(A, X)$ by

$$
(\Phi g)\left(a_{1}, \ldots, a_{p}\right)=\sum a_{1}^{0} \ldots a_{p}^{0} g\left(a_{1}^{1}, \ldots, a_{p}^{1}\right)
$$

writing the comodule structure indices as superscripts, $\Delta a_{i}=\sum a_{i}^{0} \otimes a_{i}^{1}$.

Lemma 5.6. $\Phi g \in{ }^{p} N^{p}(A, X)$ and $d_{A}(\Phi g) \in{ }^{p+1} N^{p+1}(A, X)$.

Proof. For $b \in B$, we have

$$
\Phi g\left(b a_{1}, \ldots, a_{p}\right)=\sum b a_{1}^{0} \ldots a_{p}^{0} g\left(a_{1}^{1}, \ldots, a_{p}^{1}\right)
$$

since $\Delta_{A}\left(b a_{1}\right)=\sum b a_{1}^{0} \otimes a_{1}^{1}$. Thus condition (5.1) is satisfied. Also,

$$
\begin{aligned}
(\Phi g)\left(a_{1}, \ldots, a_{i} b, a_{i+1}, \ldots, a_{p}\right) & \\
& =\sum a_{1}^{0} \ldots a_{i}^{0} b a_{i+1}^{0} \ldots a_{p}^{0} g\left(a_{1}^{1}, \ldots, a_{p}^{1}\right) \\
& =(\Phi g)\left(a_{1}, \ldots, a_{i}, b a_{i+1}, \ldots, a_{p}\right) .
\end{aligned}
$$


Thus (5.2) is satisfied for all $i$ and $\Phi g \in{ }^{p} N^{p}(A, X)$. Since $g\left(a_{1}^{1}, \ldots, a_{p}^{1}\right) \in X^{B}$,

$$
\begin{aligned}
(\Phi g)\left(a_{1}, \ldots, a_{p} b\right) & \\
& =\sum a_{1}^{0} \ldots a_{p}^{0} b g\left(a_{1}^{1}, \ldots, a_{p}^{1}\right) \\
& =\sum a_{1}^{0} \ldots a_{p}^{0} g\left(a_{1}^{1}, \ldots, a_{p}^{1}\right) b=\Phi g\left(a_{1}, \ldots, a_{p}\right) b
\end{aligned}
$$

and it follows that $d_{A} \Phi g \in{ }^{p+1} N^{p+1}(A, X)$.

Lemma 5.7. For $g \in{ }^{p} N\left(C, X^{B}\right), \Psi^{p 0} \Phi g=g$.

Proof. For any $\mathfrak{K}$-linear function $t: C \rightarrow X$, setting $u\left(a \otimes_{B} a^{\prime} \otimes c\right)=a a^{\prime} t(c)$ for $a, a^{\prime} \in A$ and $c \in C$ defines a $\mathfrak{K}$-linear function $u: A \otimes_{B} A \otimes C \rightarrow X$. By Lemma $2.1(\mathrm{~d})$,

$$
\begin{aligned}
\sum r(c) l(c)^{0} t\left(l(c)^{1}\right) & =u\left(\sum r(c) \otimes_{B} l(c)^{0} \otimes l(c)^{1}\right) \\
& =u\left(\sum r\left(c^{1}\right) \otimes_{B} l\left(c^{1}\right) \otimes c^{2}\right) \\
& =\sum r\left(c^{1}\right) l\left(c^{1}\right) t\left(c^{2}\right) \\
& =\sum \epsilon\left(c^{1}\right) t\left(c^{2}\right) \text { by Lemma } 2.1(\mathrm{c}) \\
& =t(c) .
\end{aligned}
$$

Using this with $t(c)=\sum l\left(c_{2}\right)^{0} \ldots l\left(c_{p}\right)^{0} g\left(c, l\left(c_{2}\right)^{1}, \ldots, l\left(c_{p}\right)^{1}\right)$, we have

$$
\begin{aligned}
\left(\Psi^{p 0} \Phi g\right)\left(c_{1}, \ldots, c_{p}\right) & =\sum r\left(c_{p}\right) \ldots r\left(c_{1}\right)(\Phi g)\left(l\left(c_{1}\right), \ldots, l\left(c_{p}\right)\right) \\
& =\sum r\left(c_{p}\right) \ldots r\left(c_{1}\right) l\left(c_{1}\right)^{0} \ldots l\left(c_{p}\right)^{0} g\left(l\left(c_{1}\right)^{1}, \ldots, l\left(c_{p}\right)^{1}\right) \\
& =\sum r\left(c_{p}\right) \ldots r\left(c_{2}\right) l\left(c_{2}\right)^{0} \ldots l\left(c_{p}\right)^{0} g\left(c_{1}, l\left(c_{2}\right)^{1}, \ldots, l\left(c_{p}\right)^{1}\right) .
\end{aligned}
$$

Repeating this argument gives the result.

Lemma 5.8. If $f \in{ }^{p} N^{p}(A, X)$ and $d_{A} f \in{ }^{p+1} N^{p+1}(A, X)$, then $\Phi \Psi^{p 0} f=f$.

Proof. Setting $u\left(a \otimes_{B} a^{\prime}\right)=\sum \operatorname{ar}\left(a_{p-1}^{1}\right) \ldots r\left(a_{1}^{1}\right) f\left(l\left(a_{1}^{1}\right), \ldots, l\left(a_{p-1}^{1}\right), a^{\prime}\right)$ defines a $\mathfrak{K}$-linear function $u: A \otimes_{B} A \rightarrow X$ by Lemma 5.3(b) and condition 5.2. We have

$$
\begin{aligned}
\left(\Phi \Psi^{p 0} f\right)\left(a_{1}, \ldots, a_{p}\right) & =\sum a_{1}^{0} \ldots a_{p}^{0}\left(\Psi^{p 0} f\right)\left(a_{1}^{1}, \ldots, a_{p}^{1}\right) \\
& =\sum a_{1}^{0} \ldots a_{p}^{0} r\left(a_{p}^{1}\right) \ldots r\left(a_{1}^{1}\right) f\left(l\left(a_{1}^{1}\right), \ldots, l\left(a_{p}^{1}\right)\right) \\
& =\sum a_{1}^{0} \ldots a_{p-1}^{0} u\left(a_{p}^{0} r\left(a_{p}^{1}\right) \otimes_{B} l\left(a_{p}^{1}\right)\right) \\
& =\sum a_{1}^{0} \ldots a_{p-1}^{0} u\left(1 \otimes_{B} a_{p}\right) \text { by Lemma } 2.1(\mathrm{~b}) \\
& =\sum a_{1}^{0} \ldots a_{p-1}^{0} 1 r\left(a_{p-1}^{1}\right) \ldots r\left(a_{1}^{1}\right) f\left(l\left(a_{1}^{1}\right), \ldots, l\left(a_{p-1}^{1}\right), a_{p}\right) .
\end{aligned}
$$

Repeating this argument gives the result.

Corollary 5.9. The $\eta^{p 0}$ are isomorphisms.

Proof. $E_{1}^{p 0}$ is the set of $f \in{ }^{p} N(A, X)$ with $d_{A} f \in{ }^{p+1} N^{p+1}(A, x)$, and $\eta^{p 0}$ is the restriction $\Psi^{p 0} \mid E_{1}^{p 0} \rightarrow{ }^{p} N\left(C, X^{B}\right)$. By Lemma 5.7 , it is surjective and, by Lemma 5.8 , it is injective. 
Theorem 5.10. Suppose $\bar{A}=A / \mathfrak{K} 1$ is projective as $\mathfrak{K}$-module and that $A / B$ is projective as left $B$-module. Then $\left(N_{A}, \Gamma, \eta, \gamma\right)$ is a spectral sequence constructor for $(\phi, \psi)$.

Proof. We have to show that the conditions (1), (2), (3) of Definition 3.1 are satisfied. Since

$$
{ }^{n} N_{A}(X)=\operatorname{Hom}\left(\otimes^{n}(\bar{A}), X\right)=\operatorname{Hom}\left(\bar{A}, \operatorname{Hom}\left(\otimes^{n-1} \bar{A}, X\right)\right)
$$

and $\bar{A}$ is $\mathfrak{K}$-projective, ${ }^{n} N$ is an exact functor. A function $f \in{ }^{n} N^{1}$ satisfies the further condition $f\left(b a_{1}, \ldots, a_{n}\right)=b f\left(a_{1}, \ldots, a_{n}\right)$ for all $b \in B$. In particular, $f\left(a_{1}, \ldots, a_{n}\right)=0$ if $a_{1} \in B$. Thus

$$
{ }^{n} N_{A}^{1}(X)=\operatorname{Hom}\left(\otimes^{n-1} \bar{A}, \operatorname{Hom}_{B}(A / B, X)\right) .
$$

Thus ${ }^{n} N_{A}^{1}$ is an exact functor. Similarly,

$$
\begin{aligned}
{ }^{n} N_{A}^{p}(X) & =\operatorname{Hom}\left(\otimes^{q} \bar{A}, \operatorname{Hom}_{B}\left(\otimes_{B}^{p} A / B, X\right)\right) \\
& =\operatorname{Hom}\left(\otimes^{q} \bar{A}, \operatorname{Hom}_{B}\left(A / B, \operatorname{Hom}_{B}\left(\otimes_{B}^{p-1} A / B, X\right)\right)\right)
\end{aligned}
$$

and by induction over $p,{ }^{n} N_{A}^{p}$ is exact. Thus condition (1) holds.

Let $Q$ be an injective $A^{e}$-module. Then $H^{n}\left(N_{A} Q\right)=0$ for $n>0$ by the usual theory of the normalised standard complex. We have to show that

$$
H^{q}\left(N_{A}^{p} Q / \bullet N_{A}^{p+1} Q\right)=0
$$

for $q>0$. But

$$
H^{q}\left(\bullet N_{A}^{p} Q / \bullet N_{A}^{p+1} Q\right)=E_{1}^{p q} N_{A}(Q) \simeq{ }^{p} N\left(C, H^{q}(B, Q)\right)
$$

by Corollary 5.9. But $H^{q}(B, Q)=0$ for $q>0$ since $Q$ is injective as $B^{e}$-module. Thus condition (2) holds.

Since $\Gamma(Y)=N(C, Y)$, condition (3) holds.

In the discussion of the filtered normalised complex in Barnes [1, Chapter IV], the corresponding extra assumption that $A / B$ be projective as right $B$-module was needed. If in the HS context, $A$ is a Hopf algebra, then it can be regarded as a $A / / B$-comodule algebra. The Guichardet filtration is not the same as that given by Hochschild and Serre, but by the result of the next section, the two filtrations give the same spectral sequence.

\section{UNIQUENESS OF THE SPECTRAL SEQUENCE}

As in Barnes [1 Chapter X], we construct for each cardinal $\alpha$, a cofree functor which, restricted to the subcategory $\mathcal{A}_{\alpha}$ of objects of cardinality less than $\alpha$, is a spectral sequence constructor. (This use of the subcategory $\mathcal{A}_{\alpha}$ is necessary because a cofree functor with injective model $M$ and injective basis $(M, U)$ only has the desired properties with respect to modules embeddable in $M$.) From the existence of this cofree functor, we deduce as in [1, Chapter X], that all spectral sequence constructors construct the same spectral sequence. We need one technical lemma to get around the difficulty caused by $C$-modules not being $A^{e}$-modules. For this, we again need the assumption that $\bar{A}$ is $\mathfrak{K}$-projective, that is, that there exists a $\mathfrak{K}$-module homomorphism $\epsilon: A \rightarrow \mathfrak{K}$ with $\epsilon(1)=1$.

Lemma 6.1. For every $C$-module $Y$, there exists an injective $A^{e}$-module $Q$ such that $Y$ can be embedded in $Q^{B}$. 
Proof. We first make $Z=\operatorname{Hom}(A, Y)$ a $C$-module by defining $(f \cdot c)(a)=f(a) c$ for $c \in C, a \in A$ and $f \in \operatorname{Hom}(A, Y)$. We construct an embedding $i: Y \rightarrow Z$ by setting $(i y)(a)=\epsilon(a) y$ for $y \in Y$ and $a \in A$. So defined, $i$ is a $C$-module homomorphism, because

$$
(i(y c))(a)=\epsilon(a) y c=(\epsilon(a) y) c=(i y)(a) c=((i y) \cdot c)(a)
$$

for $g \in G, a \in A$ and $f \in \operatorname{Hom}(A, Y)$. It is clearly injective.

Next, we use the standard embedding of $Z$ in the coinduced $C$-module $W=$ $\operatorname{Hom}_{\mathfrak{K}}(C, Z)$, defining $\sigma: Z \rightarrow W$ by setting $\sigma(z)(c)=z c$ for $z \in Z$ and $c \in C$. We now have an embedding of $Y$ in $\operatorname{Hom}(C, \operatorname{Hom}(A, Y))$. By Lemma 2.4, we have an embedding of $Y$ in $X^{B}$ where $X$ is the coinduced $A^{e}$-module $\operatorname{Hom}\left(A^{e}, Y\right)$. Taking any embedding of $X$ in an injective $A^{e}$-module $Q$, we get an embedding of $Y$ in $Q^{B}$.

We use the theory of cofree functors developed in [1, Chapter X]. Our spectral sequence constructors consist of two functors and two natural transformations instead of the single functor used in the HS context. To accommodate this, we shall say that the pair $(F, \Gamma)$ of functors, $F$ defined on $\mathcal{A}$ and $\Gamma$ defined on $\mathcal{C}$, is simple cofree on the basis $(M, U, V)$ if $F$ is cofree on $(M, U)$ and $\Gamma$ is cofree on $(\phi(M), V)$.

Theorem 6.2. Let $C$ be a Hopf algebra over $\mathfrak{K}$ and let $A$ be a right $C$-comodule algebra with $B=A^{c o C}$. Suppose $A, B$ and $C$ are $\mathfrak{K}$-projective and that $A / B$ is $C$ Galois. Let $\mathcal{A}$ be the category of left $A^{e}$-modules, $\mathcal{C}$ the category of right $C$-modules and let $\mathcal{D}$ be the category of $\mathfrak{K}$-modules. Let $\phi: \mathcal{A} \rightarrow \mathcal{C}$ and $\psi: \mathcal{C} \rightarrow \mathcal{D}$ be the functors defined by $\phi(X)=X^{B}$ and $\psi(Y)=Y^{C}$ for $X \in \mathcal{A}$ and $Y \in \mathcal{C}$. Suppose $A / \mathfrak{K} 1$ is $\mathfrak{K}$-projective and that $A$ is both left and right $B$-flat. Then, for any cardinal $\alpha$, there exists a simple cofree pair $(T, \Gamma)$ with injective model $M$ and injective basis $(M, U, V)$, and natural transformations $\eta, \gamma$ such that on the subcategory $\mathcal{A}_{\alpha}$ of objects of $\mathcal{A}$ of cardinality less than $\alpha,(T, \Gamma, \eta, \gamma)$ is a spectral sequence constructor for $(\phi, \psi)$.

Proof. By replacing $\alpha$ by a suitable larger limit cardinal, we may suppose that every object in $\mathcal{A}_{\alpha}$ has an injective resolution in $\mathcal{A}_{\alpha}$, and that every object of $\mathcal{C}_{\alpha}$ likewise has an injective resolution in $\mathcal{C}_{\alpha}$. There exists an injective module $X$ in $\mathcal{A}$ such that every object of $\mathcal{A}_{\alpha}$ can be embedded in $X$. Likewise, there exists an injective module $Y$ in $\mathcal{C}$ such that every object of $\mathcal{C}_{\alpha}$ can be embedded in $Y$. By Lemma 6.1, there exists an injective module $Q$ in $\mathcal{A}$ such that $Y$ can be embedded in $\phi(Q)$. Putting $M=X \oplus Q$, we obtain an injective module $M$ such that every module in $\mathcal{A}_{\alpha}$ has an injective resolution, all of whose terms can be embedded in $M$, and every module in $\mathcal{C}_{\alpha}$ has an injective resolution all of whose terms can be embedded in $\phi(M)$. By [1, Lemma X.3.2, p. 101], there exists a simple cofree functor $\Gamma$ from $\mathcal{C}$ to cochain complexes in $\mathcal{D}$ with basis $(\phi(M), V)$ for some injective $V$, and natural transformation $\gamma: H^{\bullet}(\Gamma) \rightarrow R^{\bullet} \psi$ which, on $\mathcal{C}_{\alpha}$, is a natural isomorphism. The construction of $T$ and the proof of the result now follows exactly as for [1, Theorem X.5.3, p. 107].

Theorem 6.3. Let $C$ be a Hopf algebra over $\mathfrak{K}$ and let $A$ be a right $C$-comodule algebra with $B=A^{c o C}$. Suppose $A, B$ and $C$ are $\mathfrak{K}$-projective and that $A / B$ is $C$-Galois. Let $\mathcal{A}$ be the category of left $A^{e}$-modules, $\mathcal{C}$ the category of right $C$ modules and let $\mathcal{D}$ be the category of $\mathfrak{K}$-modules. Let $\phi: \mathcal{A} \rightarrow \mathcal{C}$ and $\psi: \mathcal{C} \rightarrow \mathcal{D}$ be the functors defined by $\phi(X)=X^{B}$ and $\psi(Y)=Y^{C}$ for $X \in \mathcal{A}$ and $Y \in \mathcal{C}$. 
Suppose $A / \mathfrak{K} 1$ is $\mathfrak{K}$-projective and that $A$ is both left and right $B$-flat. Suppose $F=\left(F, \Gamma_{F}, \eta_{F}, \gamma_{F}\right)$ and $F^{\prime}=\left(F^{\prime}, \Gamma_{F}^{\prime}, \eta_{F}^{\prime}, \gamma_{F}^{\prime}\right)$ are spectral sequence constructors for $(\phi, \psi)$. Then $F$ and $F^{\prime}$ construct canonically isomorphic spectral sequences from the $E_{2}$-level onward.

Proof. The argument of [1, Theorem X.5.4, p. 109] applies unchanged.

\section{REFERENCES}

[1] D. W. Barnes, Spectral sequence constructors in algebra and topology, Mem. Amer. Math. Soc. 53 (1985). MR 86e:55032

[2] H. Cartan and S. Eilenberg, Homological Algebra, Princeton University Press, 1956. MR 17:1040e

[3] A. Guichardet, Suites spectrales à la Hochschild-Serre pour les produits croisés d'algèbres et de groupes, J. Algebra 235 (2001), 744-765. MR 2001m:16013

[4] H. J. Schneider, Representation theory of Hopf Galois extensions. Hopf algebras, Israel J. Math. 72 (1990), 196-231. MR 92d:16047

[5] D. Stefan, Hochschild cohomology on Hopf Galois extensions, J. Pure Appl. Algebra 103 (1995), 221-233. MR 96h:16013

1 Little Wonga Road, Cremorne NSW 2090, Australia

E-mail address: donb@netspace.net.au 